Strafgesetzbuches von 1926 erfolgte Änderung des Strafensystems hervor. Vierundsiebzig Peitschenhiebe werden als häufige Strafe verankert (S. 23), im übrigen auch für Frauen, die sich in der Öffentlichkeit ohne vorschriftsmäßige Kleidung (d. h. unverschleiert) zeigen (Art. 102).

Ein Blick in die Strafgesetze der Islamischen Republik Iran, den die Autorin durch ihre Veröffentlichung in deutscher Sprache ermöglicht, sollte nicht nur für den Juristen und Strafrechtler, sondern auch für denjenigen von Interesse sein, der sich mit Religion und Gesellschaft Irans beschäftigt. Ihr gebührt der Dank für eine rundum gelungene Arbeit. Der Leser darf auf ihre zukünftigen Veröffentlichungen gespannt sein.

Hans-Georg Ebert

\title{
Florian Amereller
}

Hintergründe des "Islamic Banking". Rechtliche Problematik des riba-Verbotes in der Sharia'a und seine Auswirkungen auf einzelne Rechtsordnungen arabischer Staaten Schriften zum Internationalen Recht, Bd. 71

Duncker \& Humblot Verlag, Berlin, 1995, 206 S., DM 112,--

Die Rolle des islamischen Rechts ist in Handelsbeziehungen mit arabischen Ländern, vor allem mit den Golfstaaten, ein großer Unsicherheitsfaktor. Viele international übliche Vertragsklauseln können vor dem klassischen islamischen Recht nicht bestehen. Stolpersteine bilden vor allem das Verbot von gharar, was etwa mit "Unsicherheit" oder "Unbestimmtheit" übersetzt werden kann, und von riba, was gemeinhin unscharf im Sinne von "Zins" oder "Wucher" verstanden wird. Die Staaten der islamischen Welt stehen vor dem Problem, religiös fundierte Normen mit den Anforderungen des heutigen Wirtschaftslebens zu versöhnen. Das Problem wird noch dadurch verschärft, daß die Aussagen des klassischen islamischen Rechts gerade in diesen Bereichen alles andere als eindeutig sind. Ihre zentrale Stellung im Islam macht sie jedoch zu einem Kristallisationspunkt von Reislamisierungsbestrebungen, die immer auch mit einer Abwehr tatsächlicher oder vermeintlicher Überf remdung einhergehen.

Amerellers Arbeit, eine Mainzer juristische Dissertation, hat sich zum Ziel gesetzt, das riba-Verbot darzustellen und seinen Einfluß auf das heutige Zivilrecht arabischer Staaten zu untersuchen. Ein solches Vorhaben setzt nicht nur juristische Vorbildung, sondern auch erhebliche Kenntnisse in Sprache und Kultur voraus und ist schon deswegen anerkennenswert. Durch den aktuellen Bezug ist sie darüber hinaus auch praxisrelevant und verspricht Aufschlüsse über das Kräfteverhältnis von, grob gesagt, Islamisierungs- und Säkularisie- 
rungstendenzen in den einzelnen arabischen Staaten. Eine derartige Monographie liegt in deutscher Sprache bislang noch nicht vor. ${ }^{1}$

Die Arbeit ist in sieben Kapitel nebst einer Einleitung untergliedert. Das 1. Kapitel "Shari'a und Zivilrecht - Gesetz, statisches System oder moralischer Kodex?" (S. 20-43) enthält allgemeine Aussagen über Wesen, Werden und aktuelle Relevanz des islamischen Rechts in den arabischen Staaten. Was der Verfasser hier bringt, ist nicht neu und beruht vor allem auf einer - bisweilen etwas unkritischen - Auswertung der Sekundärliteratur. Der Verfasser übernimmt die mittlerweile nicht mehr ganz unangefochtene These der westlichen Orientalistik, daß das islamische Handelsrecht im wesentlichen toter Buchstabe gewesen sei (S. 25 f.). Dennoch schließt er sich wenig später der Aussage an, das riba-Verbot habe "maßgeblich die Entwicklung eines kapitalistischen Systems nach westlichem Muster in den arabischen Staaten verhindert" (S. 40). Ein Produkt des riba-Verbotes sind die Bemühungen, ein Banksystem nach islamischen Grundsätzen zu schaffen, die sich im Zuge der Reislamisierung verstärkten (s. 40 ff.). Für das Verständnis des riba-Verbotes ist es wichtig, darauf hinzuweisen, daß dieses Verbot zusammen mit dem gharar-Verbot die Vertragsfreiheit in einem Maße einschränkt, die weit über ein bloßes Zinsverbot hinausgeht und Verträge aller Art erfassen kann (S. 33-37). Allerdings sind die Ausführungen zu dogmatischen Einzelheiten für jemanden, der die erst im zweiten Kapitel folgenden Ausführungen zum riba-Verbot noch nicht gelesen hat, sicherlich nicht ganz leicht nachzuvollziehen.

Im zweiten Kapitel werden sich der Verfasser dann dem "Riba in der klassischen islamischen Lehre" zu (S. 44-71). Auch hier beruhen seine Aussagen im wesentlichen auf Sekundärliteratur, daneben zieht er immer wieder auch klassische Werke heran (allerdings ohne seine Auswahl zu begründen). Einleitend weist der Verfasser darauf hin, daß der Begriff riba nicht übersetzbar ist, daß jedenfalls eine Übersetzung mit "Zins" oder "Wucher" viel zu kurz greifen würde (S. 46). Die Aussagen des Koran sind nicht eindeutig, ergiebiger sind verschiedene Überlieferungen vom Propheten (sunna), die im islamischen Recht im Rang gleich nach dem Koran kommen. Dennoch ist es den islamischen Juristen nicht gelungen, die Reichweite des riba-Verbotes eindeutig zu bestimmen. Viele Fragen bleiben offen. Deutlich wird jedoch, daß die Überlieferungen viel häufiger Tausch- und Kaufverträge betreffen als Darlehen. Der Verfasser stellt die verschiedenen Positionen im wesentlichen richtig dar. Störend wirkt es nur, wenn er für Aussagen zum klassischen islamischen Recht nur auf westliche Sekundärliteratur oder moderne Interpreten verweisen kann (so etwa auf S. 58 in den Fußnoten 82 und 87).

Das dritte Kapitel behandelt unter der Überschrift "Moderne Interpretationsansätze" (S. 7396) Bemühungen moderner Gelehrter (deren Lebensdaten der Verfasser allerdings verschweigt), das einerseits rigorose, andererseits nur schwer greifbare riba-Verbot so zu interpretieren, daß es den Bedürfnissen der Zeit, wie sie sie sahen, entspricht. Zunächst

Vgl. aus dem englischen Sprachraum etwa W.M. Ballantyne, Commercial Law in the Arab Middle East: The Gulf States; S.E. Rayner, The Theory of Contracts in Islamic Law, 1991; N. Saleh, Unlawful Gain and Legitimate Profit in Islamic Law, 1986. 
geht der Verfasser allerdings auf Dissidenten und Praktiken früherer Jahrhunderte ein, die Ansätze für eine Reinterpretation des riba-Verbotes bieten (S. 73-82). Die neueren Ansätze laufen im wesentlichen darauf hinaus, moderate Zinsen ohne Zinseszinsen in investiven, nicht jedoch in konsumtiven Darlehen zu erlauben. Das erfordere das "öffentliche Wohl" wegen der mittlerweile zentralen Funktion des zinsbringenden Darlehens in den modernen Wirtschaftsbeziehungen.

Im anschließenden 4. Kapitel "Möglichkeiten des ijtihad und der Umgehung des ribaVerbotes" (S. 97-109) werden dann dogmatische Ansätze untersucht, mit deren Hilfe dem riba-Verbot in der Praxis die Schärfe genommen werden konnte und kann. Erst damit wird die Tragweite, aber auch die Problematik der im vorigen Kapitel behandelten "modernen Interpretationsansätze" erfaßbar. Der Raum für offene Rechtsfortbildung durch individuelle Rechtsgelehrte ist im islamischen Recht dort, wo es sich auf Offenbarung gründet, natürlich sehr beschränkt. Wegen der "Signalfunktion des riba-Verbotes für den Islam" (S. 98) ist sie gerade in diesem Bereich kaum denkbar. Abweichende Interpretationen der autoritativen Quellen haben allenfalls "persuasive authority" und können erst dann, wenn sie sich zum allgemeinen Konsens (igma') verdichtet haben, in das Gebäude des islamischen Rechts Eingang finden. Deshalb ist die Durchschlagskraft von Argumenten etwa aus dem öffentlichen Interesse (maslaha) oder mit den Mitteln einer teleologischen Reduktion (istihsan) gering. Der Verweis auf die schiere Notwendigkeit (haja, darura) kann nur in Einzelfällen Ausnahmen rechtfertigen, nicht jedoch eine generell abweichende Regelung. Nicht einmal im Verhältnis zu Nichtmuslimen läßt das islamische Recht echten Spielraum für Zinsvereinbarungen. Trotz oder gerade wegen dieser Schwierigkeit boten einige Schulen des islamischen Rechts schon seit der Frühzeit Möglichkeiten an, das riba-Verbot im Wege einer rein formalen Interpretation zu umgehen. Die Ausführungen des Verfassers zu diesen Umgehungsgeschäften (hiyal) bleiben allerdings sehr oberflächlich (S. 106-109).

Im folgenden, dem 5. Kapitel ("Darlehen und Finanztransaktionen nach islamischem Recht", S. 110-132) untersucht der Verfasser, welche Alternativen sich Banken bieten, die dem riba-Verbot genügen wollen. Im Kern geht es natürlich darum, Zinsen zu vermeiden. Der Verfasser behandelt von den "islamischen Bankgeschäften" den nur zinslos zulässigen und jederzeit widerruflichen Kredit (qard), für den in der Praxis häufig "Gebühren" erhoben werden, weiter die in der Praxis seltene Beteiligungsgesellschaft (musharaka) sowie die "stille Gesellschaft" (mudaraba), deren Bedeutung für die Theorie eines islamischen Bankwesens wesentlich größer ist als für die Praxis, und schließlich den islamischrechtlich umstrittenen, praktisch jedoch besonders bedeutsamen Weiterverkauf mit Gewinnangabe (murabaha). Diese Transaktionen sind zumeist entweder im Wirtschaftsleben nicht konkurrenzfähig oder können vor dem islamischen Recht nicht bestehen. Für seine Darstellung stützt sich der Verfasser im wesentlich auf Sekundärliteratur, dabei gelingt es ihm leider nicht immer, die praktische Relevanz der verschiedenen Vertragsgestaltungen deutlich zu machen. ${ }^{2}$

Vgl. dazu aus wirtschaftswissenschaftlicher Sicht Grahammer, Islamische Banken. Ausweg aus dem Finanzierungsdilemma für Nahostgeschäfte, 1993. 
Besonders interessant ist das 6. Kapitel ("Zinsen und riba in einigen arabischen Staaten", S. 133-174), in dem der Verfasser die praktische Behandlung von vertraglichen Zinsvereinbarungen in einigen arabischen Staaten darstellt. Besonders eingehend wird die Lage in den Vereinigten Arabischen Emiraten behandelt (S. 133-149), einen weiteren Schwerpunkt bildet Saudi-Arabien (S. 149-161), daneben finden auch Kuwait (S. 161-166), Bahrain (S. 166-168), Oman (S. 168-171) Qatar (S. 171-172) und Libyen (S. 173-174) Berücksichtigung. Alle Staaten haben auf verschiedenen Wegen versucht, die Bedürfnisse des internationalen Wirtschafts verkehrs, der ohne Zinsvereinbarungen kaum funktionieren würde, mit den islamischrechtlichen Anforderungen zu vereinbaren. Meist werden Handelsverträge direkt oder verdeckt dem Anwendungsbereich des riba-Verbotes entzogen. Vor allem, wenn das verdeckt geschieht, wie etwa in Saudi-Arabien, geht das auf Kosten der Rechtssicherheit. Es erscheint bislang offenbar unmöglich, islamisches Recht und Wirtschaftsleben zu versöhnen.

Abgerundet wird das Werk durch ein arabisch-deutsches Glossar und ein recht ausführliches Literaturverzeichnis. Leider fehlt ein Stichwortverzeichnis, das die Arbeit auch als Nachschlagewerk verwendbar gemacht hätte. Das Werk füllt eine Lücke. Meine kritischen Anmerkungen sollen seine Bedeutung nicht schmälern. Für Praktiker wird vor allem die Darstellung der Rechtslage in einigen arabischen Staaten interessant sein, die durchweg wichtige Handelspartner Deutschlands sind. Jedem, der sich wissenschaftlich mit aktuellen Problemen des riba-Verbotes beschäftigt, bietet es einen hilfreichen Einstieg in die komplexe und nur schwer durchschaubare Materie.

Johannes Christian Wichard

\section{Hans-Georg Ebert}

Das Personalstatut arabischer Länder: Problemfelder, Methoden, Perspektiven

Ein Beitrag zum Diskurs über Theorie und Praxis des islamischen Rechts

Leipziger Beiträge zur Orientforschung, Bd. 7

Peter Lang Verlag, Frankfurt am Main, 1996, 187 S., DM 65,--

Ziel des Werkes ist es, "die familienrechtlichen Bestimmungen der arabischen Länder vergleichend" zu analysieren. Einschränkend vermerkt der Autor allerdings, daß "von den 23 Mitgliedern der Arabischen Liga ... Djibouti, die Komoren, Mauretanien, Somalia, Sudan und Palästina sowie die nicht dieser Organisation angehörende Westsahara aus quellenmäßigen, staatsrechtlichen und anderen Gründen nicht oder nur teilweise erfaßt" werden (S. 9). Da die arabischen Staaten im Bereich des Familien- und Erbrechts zumeist nach Konfessionen differenzieren ( $S$. 14 ff.), beschränkt sich die Arbeit auf die für die sunnitischen Muslime geltenden Vorschriften. Auch dann bleibt noch genügend Material, um Bibliotheken zu füllen. Eine Arbeit, die diese Materialfülle sichtet und knapp zusam- 